\title{
A study of water exchange in wood by means of 2D NMR relaxation correlation and exchange
}

\author{
Julia Cox ${ }^{1}$, Peter J. McDonald ${ }^{1, *}$ and Barry \\ A. Gardiner ${ }^{2}$ \\ ${ }^{1}$ Department of Physics, University of Surrey, Guildford, \\ Surrey, UK \\ ${ }^{2}$ Forest Research, Northern Research Station, Roslin, \\ Midlothian, UK \\ *Corresponding author. \\ Department of Physics, University of Surrey, Guildford, \\ Surrey GU2 7XH, UK \\ E-mail: p.mcdonald@surrey.ac.uk
}

\begin{abstract}
Two-dimensional $T_{1}-T_{2}{ }^{1} \mathrm{H}$ NMR relaxation correlation spectra and $T_{2}-T_{2}$ relaxation exchange spectra for samples of spruce wood across the full moisture content range from $0 \%$ to $>100 \%$ are presented. The $T_{1}-T_{2}$ spectra unambiguously identify water in different environments within the wood that overlap in the corresponding and more traditional one-dimensional $T_{1}$ or $T_{2}$ experiment. The $T_{2}-T_{2}$ spectra unambiguously reveal the presence of water exchange between the lumen and cell wall above the fibre saturation point in sapwood. An estimate is made of the exchange rate: $1 / 30$ to $1 / 3 \mathrm{~ms}^{-1}$.
\end{abstract}

Keywords: exchange; nuclear magnetic resonance; relaxation; spruce; water.

\section{Introduction}

Wood is a complex biological material with non-uniform physical, chemical and morphological characteristics (Salmén and Burgert 2008). It is an important material in a wide range of industries that include building and construction, paper production, and increasingly cultivation of biomass for energy generation. The in-use properties of wood depend both on its microstructure and water content. In the green state, wood has a high water content which can be characterized by ${ }^{1} \mathrm{H}$ NMR. Parameters typically measured include the ${ }^{1} \mathrm{H}$ nuclear spin-relaxation times of water-hydrogen components in the sample: the transverse relaxation time $T_{2}$ and the longitudinal relaxation time $T_{1}$. These parameters depend on the local environment of the water proton and are a reflection of its mobility.

Numerous studies of one-dimensional (1D) relaxation time distributions in wood (Nanassy 1973, 1974; Riggin et al. 1979; Byrne et al. 1986; Araujo et al. 1992; Hartley et al. 1994; Fantazzini et al. 2006; Labbe et al. 2006; Ahmeida et al. 2007; Thygesen and Elder 2008) revealed multi-modal distribution of relaxation times representing water in different structural environments. These typically include a slowly relaxing component $(\sim 100 \mathrm{~ms})$ characteristic of the lumen water within cells that is seen only above fibre saturation point (FSP) and one or more intermediate relaxing components ( $\sim 1-10 \mathrm{~ms})$ assigned to water in cell walls. This component can be divided into water adsorbed on internal surfaces, water in clusters and to hydrogen bonds in plasticised wood polymer. Finally, given a sufficiently high spectrometer specification, a very fast relaxing $T_{2}$ component $(\sim 10 \mu \mathrm{s})$, characteristic of hydrogen in solid wood is observed. In spruce, as investigated in this study, tracheids are the dominant cell structures and make up over $95 \%$ of the wood cells. They are between 1 and $4 \mathrm{~mm}$ long and 10 and $60 \mu \mathrm{m}$ wide in Sitka spruce [Picea sitchensis (Bong.) Carr.] (Mitchell and Denne 1997; Macdonald and Hubert 2002) and Norway spruce [Picea abies (L.) Karst.] (Havimo et al. 2008).

In conventional $1 \mathrm{D}$ relaxation analyses, exchange can be inferred from an observed "averaging" of relaxation rates (Zimmerman and Brittin 1957), and hence a subset of these studies has examined the possibility of exchange of water between the different components. Nanassy (1974) concluded that a substantial fraction of "wood" hydrogen (presumably hydroxyls in non-crystalline structures) can exchange with "water"' hydrogen. Riggin et al. (1979) inferred an exchange rate for hydrogen between the solid wood and cell wall adsorption sites of $0.35 \mathrm{~ms}^{-1}$ and between the cell wall and free water of $0.09 \mathrm{~ms}^{-1}$ by carefully measuring the ratio of signal component amplitudes assigned to different reservoirs and their respectively changing relaxation times as a function of moisture content (MC), although with some caveats related to the interdependence of data fitting parameters. Hartley et al. (1994) similarly calculated an exchange time between water and wood of $0.1 \mathrm{~ms}^{-1}$, but importantly noted that the interpretation was not unambiguous and that alternate interpretations of the data involving the plasticisation of the wood polymer were possible. Fantazzini et al. (2006) carried out a comprehensive study of eight wood types and inferred exchange in six of these woods with comparable exchange rates to those quoted above.

Two-dimensional (2D) relaxation experiments offer an enhancement to basic 1D experiments (Peemoeller et al. 1981; Song et al. 2002). The basis of a 2D experiment is that the relaxation time is measured twice in quick succession. In the $T_{1}-T_{2}$ correlation experiment, first the $T_{1}$ relaxation is encoded for a period $\tau_{1}$ and then the $T_{2}$ relaxation for a period $\tau_{2}$. This yields a dataset $s\left(\tau_{1}, \tau_{2}\right)$. The 2D inversion produces $S\left(T_{1}, T_{2}\right)$, the correlation map of $T_{1}$ against $T_{2}$. 
Distinct reservoirs of ${ }^{1} \mathrm{H}$ (i.e., water) are revealed as islands in this map. The $T_{2}-T_{2}$ exchange experiment is similar except that $T_{2}$ is measured in both intervals and a short period (exchange interval) is left between the separate encodings during which the signal is stored. Most reservoirs are now revealed as diagonal features on the $2 \mathrm{D}$ map. The $T_{2}$ measured in the first interval is the same as that measured in the second. There is no additional information compared with the $1 \mathrm{D}$ experiment. However, this is not true for any ${ }^{1} \mathrm{H}$ that changes environment during the storage interval. This environment exchanging ${ }^{1} \mathrm{H}$ is detected as an off-diagonal peak.

The advantages of $2 \mathrm{D}$ experiments are clear. First, overlapping peaks in a $1 \mathrm{D}$ (either $T_{1}$ or $T_{2}$ ) relaxation time distribution - arising from water in different components but with similar relaxation times - can be well resolved in the 2D $T_{1}-T_{2}$ correlation. This can remove ambiguities and hence significantly assist in the assignment of features. Second, exchange can be unambiguously identified in the $T_{2}-T_{2}$ exchange experiment. Moreover, by making measurements as a function of exchange time, exchange rates can be inferred in a single sample without recourse to measurements at, for instance, multiple MCs or without recourse to a priori knowledge of relaxation times in the absence of exchange.

Although the opportunity for these experiments has been realised for many years, their interpretation relies on a $2 \mathrm{D}$ Laplace inversion and it is only in recent years that a sufficiently reliable and robust algorithm has been developed for routine use (Venkataramanan et al. 2002).

Several authors are now applying $T_{1}-T_{2}$ and $T_{2}-T_{2}$ experiments to characterise a range of condensed matter including rocks (Song et al. 2002; Washburn and Callaghan 2006) and ceramics (Casieri et al. 2009), and in food (Marigheto et al. 2008) and myocardial tissue (Seland et al. 2007). Recent advances have been reviewed by Callaghan et al. (2007). We have previously used $T_{1}-T_{2}$ and $T_{2}-T_{2}$ experiments for a detailed analysis of pore-water dynamics within the microstructure of cement pastes (McDonald et al. 2005; Monteilhet et al. 2006) revealing exchange between nano- and mesoscale porosities. Within the context of this study, we also performed a detailed analysis of the $2 \mathrm{D}$ spectrum across a broad range of relaxation and exchange regimes.

The authors are aware of only three previous attempts to measure 2D relaxation spectra in wood. Prior to the availability of the robust Laplace inversion algorithm by Venkataramanan et al. (2002), Xu et al. (1996) measured $T_{1}-T_{2}$ correlations in red cedar wood. Clear spectral features are observed but the data are of very low signal-to-noise $(\mathrm{S} / \mathrm{N})$ ratio, and although exchange is discussed no exchange peaks are observed. More recently, Mikac et al. (2007) and Van As et al. (2008) presented preliminary 2D relaxation spectra recorded from wood samples with the latter reporting features in the $T_{1}-T_{2}$ spectra tentatively assigned to exchange but without quantitative analysis.

The purpose of this study is two-fold. First, we attempt to separate into different compartments water which in $1 \mathrm{D}$ $T_{1}$ or $T_{2}$ measurements is manifest with a single relaxation time. The experiments should be performed quantitatively across the full MC range from dry to green wood. Thereby, a better discrimination of water is sought than has hitherto been possible. This is achieved primarily by measurement of $T_{1}-T_{2}$ relaxation correlation spectra. The second objective is to observe exchange of water between these different compartments and to directly measure the exchange rates. Aiming at this, $T_{2}-T_{2}$ relaxation exchange spectra are measured.

\section{Methods}

The instrument employed for ${ }^{1} \mathrm{H}$ NMR measurements at room temperature was a Maran benchtop NMR spectrometer (Oxford Instruments, Oxon, UK) operating at $20 \mathrm{MHz}$. The $T_{1}-T_{2}$ correlation and $T_{2}-T_{2}$ exchange experimental pulse sequences are

$\left[P_{180^{-}}-\alpha^{i} \tau_{1}-P_{90}-\beta^{0} \tau_{2}-\left(P_{180^{-}}-\beta^{j} \tau_{2}-\text { echo- } \beta^{j+1} \tau_{2^{-}}\right)_{m^{-}} \tau_{R D}\right]_{n}$

and

$\left[P_{90}-\beta^{0} \tau_{2}-\left(P_{180}-\beta^{i} \tau_{2}-e c h o-\beta^{i+1} \tau_{2^{-}}\right)_{i \max }-P_{90^{-}} \tau_{e x}{ }^{-}\right.$

$\left.-P_{-90}-\beta^{0} \tau_{2}-\left(P_{180}-\beta^{j} \tau_{2}-\text { echo- } \beta^{j+1} \tau_{2}-\right)_{m}-\tau_{R D}\right]_{n}$,

respectively, as previously reported (McDonald et al. 2005; Monteilhet et al. 2006). Here $P_{90}$ is, for instance, a $90^{\circ}$ excitation pulse, $\tau$ a short delay and $\alpha^{i}$ and $\beta^{i / j}$ are factors by which the delays are logarithmically increased. In the second $T_{2}$ dimension, a total of $m=64(j=0 . .63)$ echoes were recorded spanning a $T_{2}$ encoding time range from $30 \mu \mathrm{s}$ to $1.02 \mathrm{~s}$. In the first dimension of the $T_{1^{-}}$ $T_{2}$ experiment, the $T_{1}$ delays were likewise increased logarithmically in $n=128(i=0 \ldots 127)$ steps from $0.1 \mathrm{~ms}$ to $1.0 \mathrm{~s}$. In the first dimension of the $T_{2}-T_{2}$ experiment, the encoding period spans from $60 \mu \mathrm{s}$ to $1.1 \mathrm{~s}$ in $n=128(i=0 \ldots$ imax $-1 ;$ imax $=0 \ldots n-1)$ logarithmically spaced steps. In $T_{2}-T_{2}$ experiments, the exchange period, $\tau_{e x}$ varied from 0.5 to $50 \mathrm{~ms}$. The repetition delay was typically $2.5 \mathrm{~s}$, and typically 128 , but up to 1024 , averages were acquired dependent on the observed $\mathrm{S} / \mathrm{N}$ ratio.

The 2D datasets were inverted by software running the algorithm developed by Venkataramanan et al. (2002). This algorithm has proved sufficiently stable and reliable to yield informative and meaningful datasets. However, there is a tendency for continuous relaxation time distributions to pearl, i.e., to split into a clear multimodal distribution, and for small peaks to be suppressed and/or drift towards larger peaks. In every case, we have added white noise of intensity comparable with the actual noise of datasets and repeatedly reanalysed to obtain an estimate of the errors in, and stability of, the analyses.

Exchange peaks can be expected in both $T_{1}-T_{2}$ and $T_{2}-T_{2}$ experiments. It has been shown elsewhere (McDonald et al. 2005) that, even if exchange is ongoing, for the exchange peaks to have nonzero intensity, the requirement $T_{1} \neq T_{2}$ must be met. This shifts the principal spectrum in $T_{1}-T_{2}$ experiments off-diagonal. Moreover, in $T_{1}-T_{2}$ experiments the exchange peak above the "diagonal" is negatively correlated. The inverse Laplace algorithm makes the implicit assumption (not easily rectified) of positive correlations (peaks). Hence, $T_{1}-T_{2}$ spectra with significant exchange peaks appear distorted as the algorithm attempts to fit the data. For this reason, the $T_{2}-T_{2}$ experiment is preferred for the analysis of exchange because all peaks are positive. It is also preferred because the ambiguity in differentiating exchange peaks from those arising from entities with widely differing $T_{1}$ and $T_{2}$ is removed because all peaks in the $T_{2}-T_{2}$ experiment are diagonal except exchange peaks.

Wood samples were taken from disks of felled mature spruce trunks that had been kept frozen at $-20^{\circ} \mathrm{C}$ post-felling and prior to 
use. Typically, a sample of a few cubic centimetres was cut from the disc from an area of relatively homogeneous structure, free from resin canals or reaction wood. Samples were taken from both the heartwood and sapwood areas, avoiding the transition zone between the two. For the $T_{1}-T_{2}$ experiments, the wood section was cut into small sticks around the width of a matchstick and $1 \mathrm{~cm}$ in length. The sticks were variously measured at this green MC, allowed to dry to specific hydrations prior to measurement or (in the case of heartwood) hydrated to higher MC as described below. Drying was accomplished in two ways. For lower MC, samples were left in controlled humidity environments created above standard salt solutions. At higher MC, sapwood samples were air dried in the ambient environment until they reached the weight corresponding to the desired MC. Fresh heartwood samples with MC circa 30\% were first dried using salt solutions to low $\mathrm{MC}$ and analysed for $\mathrm{T}_{1}-\mathrm{T}_{2}$ along the way. After the low MC measurements, samples were rehydrated to high MC (circa 130\%) by boiling in distilled water until they sank. They were then redried to the higher MC values and measured. Prior to measurement, all samples were tightly wrapped in PTFE and placed in the bottom of a sealed test tube with excess space occupied by a glass stirring rod. All samples were allowed to equilibrate to remove MC spatial gradients prior to measurements. For the $T_{2}-T_{2}$ measurements, cylindrical shaped samples were allowed to air dry to the desired MC and then coated with a thin layer of epoxy resin to preserve the MC during the extended measurement period. All samples were weighed (mass $m_{w}$ ) prior to measurement. After NMR analysis, samples were reweighed and oven dried at $104^{\circ} \mathrm{C}$ until no further mass loss was apparent (typically 24-48 h) to determine the dry mass $\left(m_{d}\right)$ and hence the MC, defined as $\left(m_{w}-m_{d}\right) / m_{d} \times 100 \%$. In the case of samples that were epoxy coated, the weight both prior to and after the addition of the epoxy was noted. Before drying, the epoxy was relatively easily peeled off, again with the weight recorded before and after. In this way, the mass of epoxy was accounted for properly. Taking into account both mass measurement error and loss of mass during long experiments, the error in the quoted $\mathrm{MC}$ is calculated to range from $\pm 3.5 \%$ at the highest $\mathrm{MC}$ to less than $2 \%$ at the lowest.

$T_{1}-T_{2}$ and $T_{2}-T_{2}$ spectra are presented as contour plots with a common contour interval in each dataset. Peak intensities were calculated by defining common (across a dataset) regularly shaped regions around the peaks and integrating all intensities within the region. This overcame the apparent tendency of the inversion algorithm to sharpen more intense peaks and to broaden others. With irregularly shaped peaks, the $T_{1}$ and $T_{2}$ values were estimated by picking off values with a computer cursor based on an "eyeball" judgement of the peak centre (median value).

\section{Theory}

Monteilhet et al. (2006) previously presented a detailed analysis of the expected peak intensities in $2 \mathrm{D}$ relaxation experiments for a simple system of two coupled reservoirs $a$ and $b$. More recently, Choudhury and Schonhoff (2007) excellently summarised the generalised NMR exchange equations for both relaxation and diffusion. The resultant expressions are complex functions of the nuclear relaxation and exchange rates and of the reservoir volumes. In particular, the exchange peak intensities, $p^{+/}$, vary with the exchange period, $t$ (i.e., $\tau_{e x}$ ) according to

$p^{+/} \equiv P_{0} \exp \left(s^{+} t\right)\left(1-\exp \left(\left(s^{-}-s^{+}\right) t\right)\right)$

where $P_{0}$ is a constant dependent on the reservoir volumes, the spinspin relaxation rates and the exchange rates and

$$
\begin{aligned}
s^{+/-}= & -\frac{R_{a}+k_{a}+R_{b}+k_{b}}{2} \\
& \pm \frac{1}{2} \sqrt{\left(R_{a}+k_{a}+R_{b}+k_{b}\right)^{2}-4\left[\left(R_{a}+k_{a}\right)\left(R_{b}+k_{b}\right)-k_{a} k_{b}\right]} .
\end{aligned}
$$

Here, $R_{a}$ and $R_{b}$ are the spin lattice relaxation rates of water in the two reservoirs, respectively, and $k_{a}$ and $k_{b}$ are the inter-reservoir proton (water) exchange rates.

Extending our previous analysis, Eqs. (1) and (2) can be simplified under three possible regimes: (1) fast exchange, (2) slow exchange, and (3) intermediate exchange rate. In each case, the assumption is made of exchange between proton (water) reservoirs of equal volume in which case $k_{a}=k_{b} \equiv k$. Without loss of generality, we assume $R_{b}>>R_{a}$.

In the slow exchange limit, $R_{b}>>R_{a}>>k$, so that Eq. (2) reduce to $s^{+} \approx-R_{a}$ and $s^{-} \approx-R_{b}$. Consequently, the exchange peak intensities in the $T_{2}-T_{2}$ exchange spectra vary with storage time, $t$, as

$p^{+/-} \approx P_{0} \exp \left(-R_{a} t\right)(1-\exp (-\Delta R t))$

where $\Delta R=R_{b}-R_{a}$. This regime is thus largely insensitive to the value of $k$, except that it is small.

A similar analysis of the fast exchange limit, $k>>R_{b}>>R_{a}$ yields $s^{+} \approx-\left(R_{a}+R_{b}\right) / 2 \equiv-\bar{R}$ and $s^{-} \approx-2 k$ so that

$p^{+/} \approx P_{0} \exp (-\bar{R} t)(1-\exp (-2 k t))$.

The intermediate regime is characterised by $R_{b}>>k>>R_{a}$. Under these conditions, simplification of the equations leads to

$s^{+} \approx-\frac{R_{b} k}{\left(R_{b}+2 k\right)}$ and $s^{-} \approx-\left(R_{b}+2 k\right)$

and hence

$p^{+/-} \approx P_{0} \exp \left(-\frac{R_{b} k}{R_{b}+2 k} t\right)\left(1-\exp \left(-\frac{\left(R_{b}+2 k\right)^{2}-R_{b} k}{R_{b}+2 k} t\right)\right)$.

This final result can be readily inverted for $R_{b}$ and $k$ from the two rate constants, $s^{+}$and $s^{-}-s^{+}$, derived from data fitting:

$R_{b}=\frac{u \pm \sqrt{u^{2}-8 v}}{2}$ and $k=\frac{2 v}{u \pm \sqrt{u^{2}-8 v}}$

where $u=(x+y) / x y$ and $v=(x+y) / x^{2} y$ with $x=-1 / s^{+}$and $y=-1 /$ $\left(s^{-}-s^{+}\right)$. A real solution to first order of this approximation requires that $R_{b}>2 k$.

\section{Results}

Figure 1a shows a series of three exemplars of $T_{1}-T_{2}$ correlation spectra recorded as a function of MC from spruce sapwood together with a schematic to identify the different spectrum peaks. The $\mathrm{S} / \mathrm{N}$ ratio of the raw (pre-inversion) dataset varies from 780 (at $94 \% \mathrm{MC}$ ) to 300 (15\% MC). The characteristic features in fresh wood are a major peak high on, but just below, the diagonal $T_{1}=T_{2}$. This peak, labelled $A$, is by far the most intense at high MC. It decreases in intensity as the water content in the sample is reduced. There is a smaller peak lower down (and still just below the diagonal) labelled $C$. It is accompanied by an intermediate peak, 

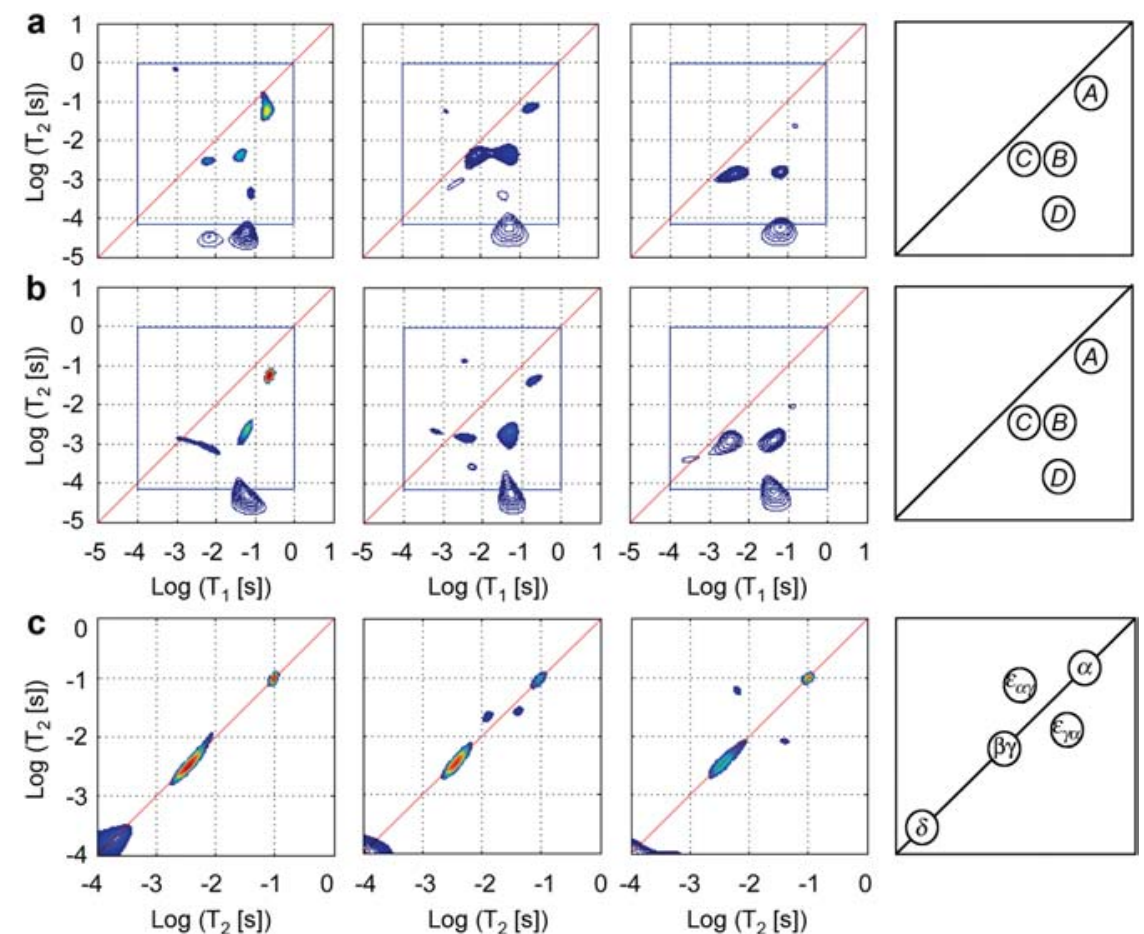

Figure 1 (a) A series of $T_{1}-T_{2}$ correlation spectra recorded from spruce sapwood at $94 \%, 44 \%$ and $15 \%$ moisture content from left to right. The spectra are shown on directly comparable intensity grey/contour scales. The inner box spans the region defined by the minimum and maximum sampled echo and relaxation delay times. A schematic identifying the primary spectral features discussed in the text is shown on the right. (b) As for (a) but for spruce heartwood at 101\%, 45\% and 14\% moisture content. (c) As for (a) but for $T_{2}-T_{2}$ exchange from a spruce sapwood sample at $45 \%$ moisture content and with exchange delays of $0.7,3$ and $12 \mathrm{~ms}$ from left to right.

$B$ to its right that has similar $T_{2}$ but longer $T_{1}$. Finally, there is a further peak below $B$, labelled $D$ that has much shorter $T_{2}$ than all the rest, but similar $T_{1}$ to $B$. The resolution of peaks $B$ and $C$ and $B$ and $D$ is an advantage of the 2D experiment. In previous 1D $T_{2}$ measurements, $B$ and $C$ overlapped, whereas in $T_{1}$ measurements the overlapping of $B$ and $D$ severely complicated any assignments. All other peaks are generally small, variable in appearance and are attributed to artefact arising from the inversion, as is the fact that more intense peaks often appear narrower than expected.

Figure 2a brings together the results of many $T_{1}-T_{2}$ experiments for spruce sapwood by showing how the intensity of peaks $A, B$ and $C$ varies as a function of MC. All intensities have been normalised to the dry mass of the sample but are otherwise in arbitrary units. Likewise, Figure $2 b$ shows the variation of the relaxation time $T_{2}$ as determined from the estimated centre of the peaks. Figures $1 \mathrm{~b}$ and $2 \mathrm{c}$ illustrate findings in the same manner as in Figures 1a and $2 \mathrm{a}$ but for spruce heartwood. The specific differences between sapwood and heartwood will be discussed in the next section.

Figure 1c shows an example of $T_{2}-T_{2}$ exchange spectra obtained with exchange periods of $0.7,3$ and $12 \mathrm{~ms}$, recorded from spruce sapwood with a MC of $45 \%$. There are two clear spectral features that lie primarily on the diagonal and that exhibit $T_{2}$ values that suggest a correspondence to first peak $A$ and second peaks $B$ or $C$. Hence, they are labelled $\alpha$ and $\beta \gamma$, respectively. There are two small features that lie symmetrically above and below the diagonal. These cannot cor- respond with the previously observed peaks. They are attributed to exchange between $\alpha$ and $\beta \gamma$ and thus labelled $\varepsilon_{\alpha \gamma}$ and $\varepsilon_{\gamma \alpha}$. The intensity in the lower left of the plot, labelled $\delta$, probably corresponds to peak $D$ in the $T_{1}-T_{2}$ spectra (with a small contamination from the epoxy resin).

Figure 3a shows the combined integrated intensity of the off-diagonal peaks, $\varepsilon_{\alpha \gamma}$ and $\varepsilon_{\gamma \alpha}$, in the $T_{2}-T_{2}$ spectrum as a function of exchange time. The error bars are estimated by adding white noise, comparable with that observed, to the data prior to Laplace inversion and repeating the inversion process. Here, the data are fitted twice according to Eq. (1). One fit assumes a baseline offset (see next section). In Figure $3 \mathrm{~b}$, the intensity of peak $\beta \gamma$ is plotted as a function of storage delay again with a fit to the data (see next section).

Measurements made of the $T_{2}-T_{2}$ exchange spectra in heartwood appeared to show significant differences to those obtained in sapwood. However, the heartwood results are difficult to interpret because of poor $\mathrm{S} / \mathrm{N}$ ratios and thus further work is required to substantiate and understand the observed differences.

\section{Analysis and discussion}

\section{$T_{1}-T_{2}$ correlation spectra and feature assignment}

The open circles in Figure 2a (sapwood) elucidate the total NMR intensity measured in the spectra correlates well with 

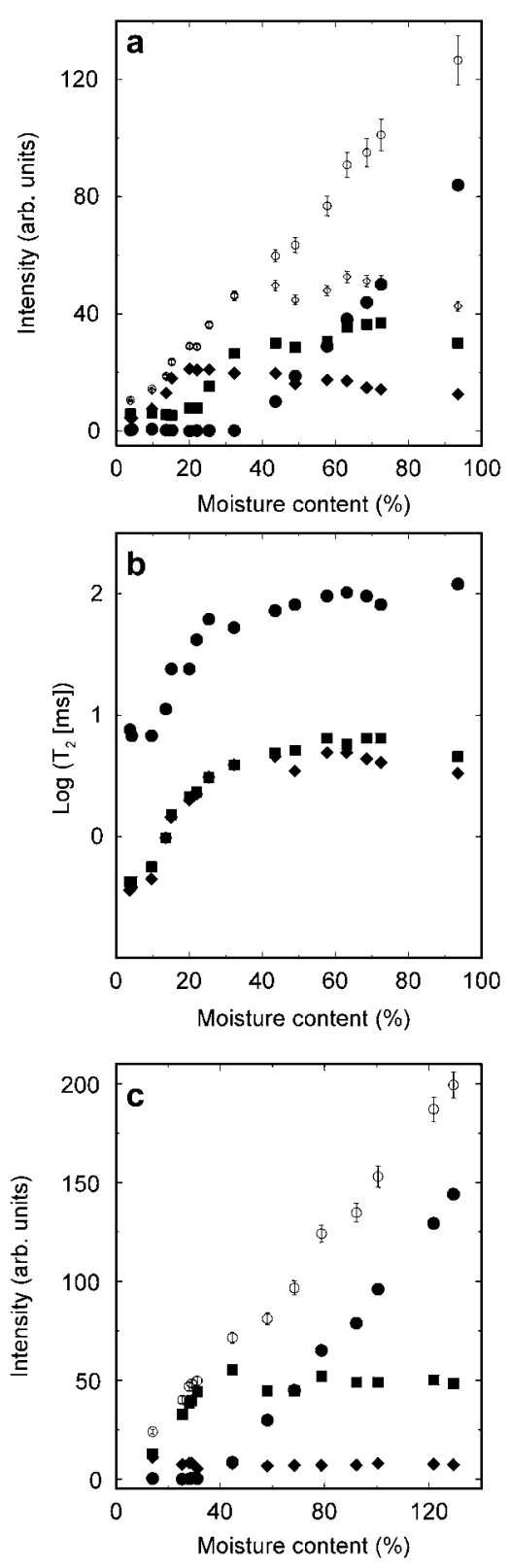

Figure 2 (a) The integrated intensity of peaks $A$ (filled circles), $B$ (filled squares) and $C$ (filled diamonds) for spruce sapwood as a function of moisture content. The open circles are the sum of the three, showing linear behaviour; the open diamonds, the sum of $B$ and $C$ which is only linear up to the FSP and thereafter constant. (b) The central $T_{2}$ relaxation time for the spruce sapwood in (a) for peaks $A$ (circles), $B$ (squares) and $C$ (diamonds) as a function of moisture content. (c) The integrated intensity of peaks $A$ (filled circles), $B$ (filled squares) and $C$ (filled diamonds) for spruce heartwood as a function of moisture content. The sum of the three is denoted by open circles.

the gravimetric MC except at the very lowest MC region (where baseline noise is high, see next section). Accordingly, the overwhelming majority of the signal is associated with evaporable water. Moreover, the open diamonds show that as MC is increased below FSP, magnetisation intensity grows in peaks $B$ and $C$, whereas above FSP it grows in peak $A$.
There is strong evidence that peak $A$ arises from water in lumen. Lumen water is the first to be removed upon drying and is only present above FSP. The peak position close to the line $T_{1}=T_{2}$ is characteristic of water in moderately large reservoirs and the relaxation times of the order of 100$200 \mathrm{~ms}$ correspond well to the long component detected by 1D measurements and generally assigned by others to lumen water. Moreover, the reduction in the relaxation time as the MC decreases (Figure 2b, circles) has also been observed in 1D measurements (Riggin et al. 1979; Araujo et al. 1992; Hartley et al. 1992). These authors have variously attributed the decrease to the changing size of the water reservoirs in the wood or, perhaps less likely, to the effects of averaging as a result of exchange. However, no entirely consistent picture has yet emerged in the literature. The dependence of the peak $A$ intensity on MC (Figure 2a, filled circles) is also consistent with lumen water. The amplitude decreases linearly with MC down to practically zero at the FSP, entirely consistent with the loss of evaporable water. In other studies, it has been thought that lumen water exhibits a range of $T_{2}$ values spanning tens to hundreds of milliseconds, representing a range of tracheid diameters (Araujo et al. 1992). Thus, it might have been expected that peak $A$ would show greater breadth. In some $2 \mathrm{D}$ measurements two closely positioned peaks were observed. It is possible that " pearling', the artefact by which a continuous distribution of relaxation times appears as a series of discrete features in the Laplace inversion forces a uni- or (less frequently) bi-modal distribution.

Peak $D$ is situated well below the diagonal almost outside the interrogated range of relaxation times with a relatively short $T_{2}$ and long $T_{1}$. A significant, but not quantified, fraction of this peak persists in experiments carried out on samples after $\mathrm{D}_{2} \mathrm{O}$ exchange. These features are characteristic of immobile, solid-like or bound ${ }^{1} \mathrm{H}$, for instance, ${ }^{1} \mathrm{H}$ bound to the wood polymer. Thus, peak $D$ is entirely consistent with the short $T_{2}$ component seen in other studies. However, the first echo time cannot be made sufficiently short to fully interrogate this peak and it is therefore probable that in these experiments only the "tip of the iceberg" is seen, i.e., that fraction of the short $T_{2}$ component distribution with the (relatively) longest $T_{2}$ values of several tens of microseconds.

It is evident that peak $B$ is almost certainly not an exchange peak linking $C$ with $D$. If it were, then it should appear strongly in the $T_{2}-T_{2}$ experiments but, in sapwood, it does not. We therefore attribute both $B$ and $C$ to water and other hydrogen in cell walls. Certainly, the $T_{2}$ value of the order of a few milliseconds is comparable with that revealed by Riggin et al. (1979), amongst others, in 1D experiments and attributed to cell wall water. Hartley et al. (1994), in particular, commented on the possibility of multiple components within this single 1D reservoir. Now, multiple components are resolved in the present experiments. As shown in Figure $2 \mathrm{a}$, both peaks increase in intensity below FSP with peak $C$ building first. Above FSP, peak $B$ continues to increase slightly while peak $C$ decreases. However, their combined intensity remains constant: no additional water accrues in this reservoir. Peak $C$ is closer to the diagonal 

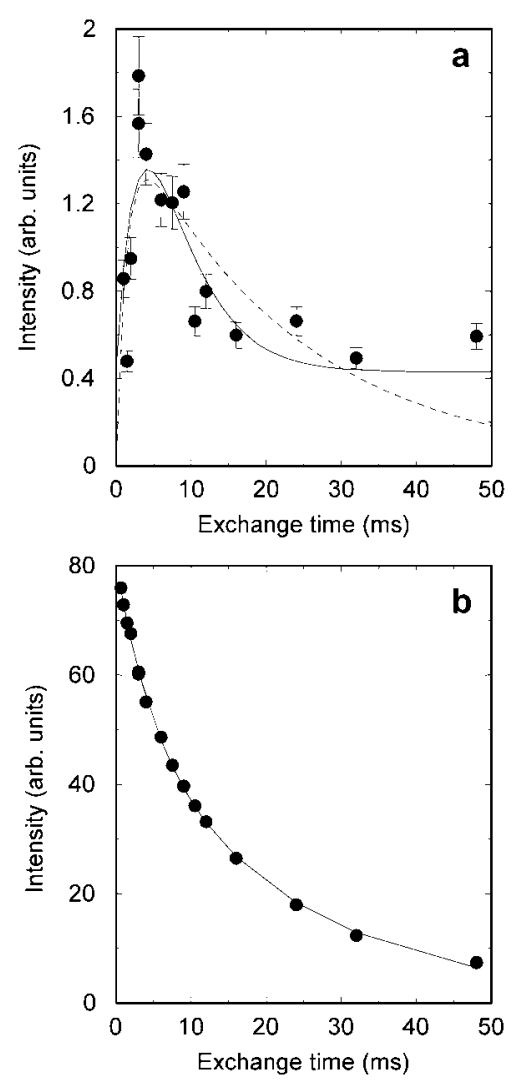

Figure 3 (a) The combined integrated intensity of peaks $\varepsilon_{\alpha \gamma}$ and $\varepsilon_{\gamma \alpha}$ in the $T_{2}-T_{2}$ exchange spectra for spruce sapwood at $45 \%$ moisture content as a function of storage delay. The solid line is a best fit to the data according to Eq. (1) with a baseline offset assumed. The dashed line is the fit without a baseline offset. Fit parameters are given in the text. (b) As for (a) but for peak $\beta \gamma$. The solid line is a two-component exponential decay least squares fit to the data. Fit parameters are given in the text.

with comparable but not equal $T_{1}$ and $T_{2}$, whereas peak $B$ has a $T_{1}$ substantially longer than $T_{2}$. Often one might assign a relaxation component with intermediate and comparable $T_{1}$ and $T_{2}$ to free but confined water such as in small clusters or voids and one with shorter $T_{2}$ and longer $T_{1}$ to more rigid or adsorbed water. However, here peak $C$ builds first as might be expected of water absorbed to wood polymer, whereas peak $B$ builds later as might be expected of free water clusters. Also consistent with this alternate scenario is the suggestion that, with the increasing development of water clusters, opportunity for direct hydrogen bonding within the wood polymer might increase. Hence, the number of accessible sites for water adsorption might decrease with a consequent decrease in the intensity of peak $C$ at higher MC as observed ${ }^{1}$.

The $T_{1}-T_{2}$ spectra recorded from heartwood are at first sight very similar to those from sapwood. Peak $B$ grows linearly below FSP and thereafter remains constant, whereas Peak A grows linearly above FSP (Figure 2c). However,

${ }^{1}$ We thank an anonymous referee for suggesting this alternate interpretation. peak $C$ retains a low value across the entire $\mathrm{MC}$ range. It is not clear why this might be. One interpretation could be that heartwood will have been naturally dried throughout its history and there are now believed to be significant differences in pore size distributions between never dried and previously dried wood (Park et al. 2006; M. Jarvis 2009, University of Glasgow, personal communication). Moreover, heartwood contains more extractives (Willför et al. 2003; Bertaud and Holmbom 2004; Xu et al. 2009) which can modify the ability of the cell wall to absorb water.

\section{$T_{2}-T_{2}$ exchange spectra: sapwood}

The $\mathrm{S} / \mathrm{N}$ ratio of the exchange peak intensity as a function of storage delay in the case of sapwood is not as good as one might wish. However, it is readily difficult to improve the situation because the experiments are already long and the wood is prone to drying throughout the course of the measurement series even with the precautions taken against this. The data show a clear trend by which the exchange peak intensity first grows and then decays away to zero as the storage time is increased. This trend enables bounds to be placed on the exchange rate.

In identifying these bounds, one should first note that peak $\beta \gamma$ in Figure 1c comprises water from the reservoirs $B$ and $C$, as suggested by the $T_{1}-T_{2}$ correlation in Figure 1a. Figure $3 \mathrm{~b}$ shows the intensity, $I$, of peak $\beta \gamma$ in the $T_{2}-T_{2}$ exchange plot together with a two component exponential decay fit: $I\left(\tau_{e x}\right)=\sum_{i=1,2} I_{i} \exp \left(-\tau_{e x} / T_{1 i}\right)$. A single component does not fit the data well. The two fit components have intensities, $I$, of 52 and 29 with relaxation times, $T_{1}$, of 23 and $4.8 \mathrm{~ms}$, respectively. The decay arises from spin-lattice relaxation in the exchange period and thus, to a good approximation, these times equate to the $T_{1}$ relaxation times of peaks $B$ and $C$ in Figure 1a. Likewise, their intensity ratio, 1.8, is comparable with the intensity ratio at similar MC of peaks $B$ and $C, 1.5$, notwithstanding that this is a different sample. It is probable that only one of these reservoirs contributes to the exchange process, most probably the peak characteristic of mobile water. The exchange time dependent relative contribution of two components to the intensity of peak $\beta \gamma$ makes it difficult to place great reliance on it in further analyses.

Second, it is noted that the intensity of peak $\alpha$ decreases throughout the experimental series (which lasted a few days) as a consequence of sample drying independent of changes in measurement exchange time. Quite deliberately, experiments as a function of exchange time were performed in pseudo-random order. This accounts for some of the apparent data scatter in Figure 3a, but not as much as might be suggested from the changing intensity of peak $\alpha$. As a consequence, it is possible that some of the water gives rise to peak $\alpha$, perhaps that which is lost first on drying. This water is not as strongly involved in the exchange process as other water. Accordingly, the intensity of peak $\alpha$ is difficult to use for further analysis of the exchange process.

An attempt was made to analyse the data in Figure 3a for information on the exchange rate. The Laplace inversion 
implicitly assumes positive intensities in the exchange spectra. Therefore, baseline noise is positive everywhere. When small peak intensities are integrated across a region, this leads to an offset with small statistical variation as the noise is thereby averaged. Hence, there is good justification for assuming a baseline offset in Figure $3 \mathrm{a}$ and an explanation for the fact that the data point error bars are small compared with the offset. Washburn and Callaghan (2006) also implicitly assumed an offset. With the offset allowed, the least squares best fit to the data, shown by the solid line in Figure 3a, yields $s^{+}=-1 /(4.5 \pm 0.3) \mathrm{ms}^{-1}$ with a reasonable degree of certainty. Approximately, $s^{+}$is the decay constant at long exchange times. The fit is much less sensitive to $\left(s^{-}-s^{+}\right)$, the rise time at short exchange times. Data fitting shows that the intensity factor, $P_{0}$, and $\left(s^{-}-s^{+}\right)$are strongly correlated. One increases as the other decreases so that the initial rise time in Figure $3 \mathrm{a}$ is ill-defined. Our best estimate yields are $\left(s^{-}-s^{+}\right)=-1 /(200 \pm 100) \mathrm{ms}^{-1}$. The best fit baseline offset is $0.43 \pm 0.01$.

The data fitting values of $\left(s^{-}-s^{+}\right)$and $s^{+}$have been used to evaluate the relaxation and exchange rates in each of the slow, intermediate and fast exchange limits by Eq. (1) and with Eqs. (3), (5) and (4), respectively. The resultant parameters are then compared with alternate estimates and tested for self-consistency. It was not possible to find a self-consistent set of parameters in the slow exchange limit based on Eq. (3).

If the fast exchange limit applies, then Eq. (4) applies so that, from the fit, $\bar{R}=1 /(4.5 \pm 0.3) \mathrm{ms}^{-1}$. If the exchange is assumed to be between peaks $A$ and $C$ in Figure 1a, then the independent experimental estimate is $\bar{R}=0.5 \times(1 / 192+$ $1 / 6.7) \approx 1 / 13 \mathrm{~ms}^{-1}$. Alternatively, if the exchange is assumed to be between peaks $A$ and $B$ in Figure 1a, then the independent experimental estimate is $\bar{R}=0.5 \times(1 / 192+1 / 47) \approx$ $1 / 75 \mathrm{~ms}^{-1}$. The former is more consistent with the $T_{2}-T_{2}$ fitting, Eq. (4), but neither is totally ruled out. The data fitting is much less sensitive to $k$ without a tight bound being placed on $\left(s^{-}-s^{+}\right)$. All reasonable unconstrained fits of the data, or of the data with possible outliers excluded, yield $\left|\left(s^{-}-s^{+}\right)\right|<1 / 15 \mathrm{~ms}^{-1}$. As a consequence, from Eq. (4), a $\max -$ imum exchange rate of the order of $k=1 / 30 \mathrm{~ms}^{-1}$ can be reasonably estimated. For exchange between peaks $A$ and $C$, this is a little less than $R_{b}$ in contravention of the basic requirement for fast exchange. However, for exchange between peaks $A$ and $B$ it is greater than $R_{b}$ in accordance with the basic requirement for fast exchange. It can be concluded with caution that the system is just, if at all, in the fast exchange regime.

The alternate option is the intermediate regime. Real values for $R_{b}$ and $k$ are not obtained for any of the fit parameter sets that include the baseline offset owing to the simplifications made. However, they can be obtained for a fit without the offset, as shown by the dotted line in Figure $3 \mathrm{a}$, albeit that this fit is of significantly poorer quality. For this fit, $s^{+}=1 / 20$ and $s_{-}^{-} s^{+}=1 / 1.5 \mathrm{~ms}^{-1}$ so that $R_{b}=1 / 1.6 \mathrm{~ms}^{-1}$ and $k=1 / 19.3 \mathrm{~ms}^{-1}$. Because $R_{a}=1 / 192 \mathrm{~ms}^{-1}$, this is in fulfilment of the intermediate exchange criterion. Based on peak $C$, the value of $R_{b}$ is a factor 4 larger ( $T_{1 b}=1.6 \mathrm{~ms}$ is smaller) than that for the $T_{1}-T_{2}$ correlation result where the $T_{1}$ is circa $6.7 \mathrm{~ms}$. Alternatively, for peak $B$, the measured and calculated $T_{1}$ values of 47 and $1.6 \mathrm{~ms}$, respectively, do not seem consistent. However, one should recall that the $T_{1}$ measurements are on different samples. Finally, if the fit without baseline is analysed according to the fast exchange limit, then $k=1 / 3 \mathrm{~ms}^{-1}$.

Probably, reality lies somewhere between the "intermediate" and "fast" regime and that an order of magnitude estimate of the exchange rate is between $1 / 30$ and $1 / 3 \mathrm{~ms}^{-1}$. The analysis has been based on a premise of equal reservoir volumes. Whilst this is an approximation, it is likely to be reasonable at a nominal MC of $45 \%$ (Figure 2a). These bounds on the exchange rate are relatively wide but consistent with previous estimates. The evidence for exchange is highly probable. To narrow this range further, data with a substantially improved $\mathrm{S} / \mathrm{N}$ ratio is needed. Moreover, emphasis should be on short storage times and data should be taken in short periods of real time so that the sample does not dry significantly during data acquisition.

\section{Conclusion}

This work is the first systematic $T_{1}-T_{2}$ correlation study of wood and presents the first $T_{2}-T_{2}$ exchange spectra for wood. The first set of experiments has distinguished the three primary hydrogen reservoirs normally seen in ${ }^{1} \mathrm{H}$ NMR studies of wood: lumen water, cell walls and wood polymer. It has unambiguously revealed that the cell wall reservoir divides into two components with comparable $T_{2}$ but different $T_{1}$. Although these components cannot yet be definitively assigned to microscopic structures within cell walls, one component has relaxation parameters consistent with mobile water and possibly water in small voids, whereas the other is more consistent with less mobile water that swells wood polymers. There are clear differences in the uptake to these reservoirs between heartwood and sapwood which can further aid interpretation in the future. The second set of experiments have identified water exchange between cell walls and lumen in the sapwood samples above FSP with an exchange rate of the order of $1 / 30$ to $1 / 3 \mathrm{~ms}^{-1}$ at circa $45 \%$ $\mathrm{MC}$.

\section{Acknowledgements}

J.C. thanks the Forest Research for financial support. The authors thank Y.-Q. Song of Schlumberger-Doll Research for use of a copy of his 2D Fast Laplace Inversion software, and Dr. M. Jarvis of the University of Glasgow for useful discussions.

\section{References}

Ahmeida, G., Gagne, S., Hernandez, R.E. (2007) A NMR study of water distribution in hardwoods at several equilibrium moisture contents. Wood Sci. Technol. 41:293-307. 
Araujo, C.D., MacKay, A.L., Hailey, J.R.T., Whittall, K.P., Le, H. (1992) Proton magnetic resonance techniques for characterisation of water in wood: application to white spruce. Wood Sci. Technol. 26:101-113.

Bertaud, F., Holmbom, B. (2004) Chemical composition of earlywood and latewood in Norway spruce heartwood, sapwood and transition zone wood. Wood Sci. Technol. 38:245-256.

Byrne, G.F., Fenn, M.D., Burgar, M.I. (1986) Nuclear magnetic resonance studies of water in tree sections. Agric. For. Meteor. 38: 307-317.

Callaghan, P.T., Arns, C.H., Galvosas, P., Hunter, M.W., Qiao, Y., Washburn, K.E. (2007) Recent Fourier and Laplace perspectives for multidimensional NMR in porous media. Magn. Reson. Imaging 25:441-444.

Casieri, C., Terenzi, C., De Luca, F. (2009) Two-dimensional longitudinal and transverse relaxation time correlation as a lowresolution nuclear magnetic resonance characterization of ancient ceramics. J. Appl. Phys. 105:034901.

Choudhury, R.P., Schonhoff, M. (2007) Pulsed field gradient NMR study of phenol binding and exchange in dispersions of hollow polyelectrolyte capsules. J. Chem. Phys. 127:234702.

Fantazzini, P., Maccotta, A., Gombia, M., Garavaglia, C., Brown, R.J.S., Brai, M. (2006) Solid-liquid nuclear magnetic resonance relaxation and signal amplitude relationships with ranking of seasoned softwoods and hardwoods. J. Appl. Phys. 100:074907.

Hartley, I.D., Kamke, F.A., Peemoeller, H. (1992) Cluster theory for water sorption in wood. Wood Sci. Technol. 26:83-99.

Hartley, I.D., Kamke, F.A., Peemoeller, H. (1994) Absolute moisture content determination of aspen wood below the fibre saturation point. Holzforschung 48:474-479.

Havimo, M., Rikala, J., Sirviö, J., Sipi, M. (2008) Distributions of tracheid cross-sectional dimensions in different parts of Norway spruce stems. Silva Fenn. 42:89-99.

Labbe, N., De Jeso, B., Lartigue, J.-C., Daudé, G., Pétraud, M., Ratier, M. (2006) Time-domain ${ }^{1} \mathrm{H}$ NMR characterization of the liquid phase in greenwood. Holzforschung 60:265-270.

Macdonald, E., Hubert, J. (2002) A review of the effects of silviculture on timber quality of Sitka spruce. Forestry 75:107-138.

Marigheto, N., Venturi, L., Hills, B. (2008) Two-dimensional NMR relaxation studies of apple quality. Postharvest Biol. Technol. 48:331-340.

McDonald, P.J., Korb, J.-P., Mitchell, J., Monteilhet, L. (2005) Surface relaxation and chemical exchange in hydrating cement pastes: a two-dimensional NMR relaxation study. Phys. Rev. E 72:011409.

Mikac, U., Sepe, A., Sersa, I., Merela, M., Oven, P. (2007) MR study of moisture content and water distribution in tree tissues. Presented at: The 9th International Conference on Magnetic Resonance Microscopy, Groupement Ampere (www.ampere. ethz.ch), Aachen, Germany.

Mitchell, M.D., Denne, M.P. (1997) Variation in density of Picea sitchensis in relation to within-tree trends in tracheid diameter and wall thickness. Forestry 70:47-60.

Monteilhet, L., Korb, J.-P., Mitchell, J., McDonald, P.J. (2006) A NMR $\mathrm{T}_{2}$-store- $\mathrm{T}_{2}$ 2-dimensional correlation relaxation study of cement pastes. Phys. Rev. E 74:061404.

Nanassy, A.J. (1973) Use of wide line NMR for measurement of moisture content in wood. Wood Sci. 5:187-193.
Nanassy, A.J. (1974) Water sorption in green and remoistened wood studied by the broad-line component of the wide line NMR spectrum. Wood Sci. 7:61-68.

Park, S., Venditti, R.A., Jameel, H., Pawlak, J.J. (2006) Changes in pore size distribution during the drying of cellulose fibers as measured by differential scanning calorimetry. Carbohyd. Polym. 66:97-103.

Peemoeller, H., Shenoy, R.K., Pintar, M.M. (1981) Two-dimensional NMR time evolution correlation spectroscopy in wet lysozyme. J. Magn. Reson. 45:193-204.

Riggin, M.T., Sharp, A.R., Kaiser, R. (1979) Transverse NMR relaxation of water in wood. J. Appl. Polym. Sci. 23:3147-3154.

Salmén, L., Burgert, I. (2008) Cell wall features with regard to mechanical performance. A review. COST Action E35 20042008: Wood machining - micromechanics and fracture. Holzforschung 63:121-129.

Seland, J.G., Bruvold, M., Brurok, H., Jynge, P., Krane, J. (2007) Analyzing equilibrium water exchange between myocardial tissue compartments using dynamical two-dimensional correlation experiments combined with manganese-enhanced relaxography. Magnet. Reson. Med. 58:674-686.

Song, Y.-Q., Venkataramanan, L., Hürlimann, M.D., Flaum, M., Frulla, P., Straley, C. (2002) $\mathrm{T}_{1}-\mathrm{T}_{2}$ correlation spectra obtained using a fast two-dimensional Laplace inversion. J. Magn. Reson. 154:261-268.

Thygesen, L.G., Elder, T. (2008) Moisture in untreated, acetylated, and furfurylated Norway spruce studied during drying using time domain NMR. Wood Fiber Sci. 40:309-320.

Van As, H., Homan, N., Windt, C., Pera, H., Vergeldt, F.J. (2008) 2D NMR approaches to characterize water dynamics, exchange and membrane permeability in biological tissue: towards imaging? Presented at: The 9th International Bologna Conference Magnetic Resonance in Porous Media. Groupement Ampere (www.ampere.ethz.ch), Boston, MA, USA.

Venkataramanan, L., Song, Y.-Q., Hürlimann, M.D. (2002) Solving Fredholm integrals of the first kind with tensor product structure in 2 and 2.5 dimensions. IEEE Trans. Signal Process. 50: 1017-1026.

Washburn, K.E., Callaghan, P.T. (2006) Tracking pore to pore exchange using relaxation exchange spectroscopy. Phys. Rev. Lett. 97:175502.

Willför, S., Hemming, J., Reunanen, M., Eckerman, C., Holmbom, B. (2003) Lignans and lipophilic extractives in Norway spruce knots and stemwood. Holzforshung 57:27-36.

Xu, Y., Araujo, C.D., MacKay, A.L., Whittall, K.P. (1996) Proton spin-lattice relaxation in wood $-T_{1}$ related to local specific gravity using a fast exchange model. J. Magn. Reson. Ser. B 110:55-64.

Xu, C., Pranovich, A., Hemming, J., Holmbom, B., Albrecht, S., Schols, H.A., Willför, S. (2009) Hydrolytic stability of watersoluble spruce O-acetyl galactoglucomannans. Holzforschung 63:61-68.

Zimmerman, J.R., Brittin, W.E. (1957) Nuclear magnetic resonance studies in multiple phase system - lifetime of a water molecule in an adsorbing phase on silica gel. J. Phys. Chem. 61: $1328-1333$.

Received April 3, 2009. Accepted October 21, 2009.

Previously published online January 14, 2010. 